\title{
Measuring proper motions of galactic dwarf galaxies with Hubble Space Telescope
}

\author{
S. Piatek ${ }^{1}$ and C. Pryor ${ }^{2}$ \\ ${ }^{1}$ Department of Physics, New Jersey Institute of Technology, \\ Newark, NJ 07102-1982, USA \\ email: piatek@physics.rutgers.edu \\ ${ }^{2}$ Dept. of Physics and Astronomy, Rutgers, the State University of New Jersey \\ 136 Frelinghuysen Rd. \\ Piscataway, NJ 08854-8019, USA \\ email: pryor@physics.rutgers.edu
}

\begin{abstract}
Over the past several years, our research group has been measuring proper motions for nearby dwarf satellite galaxies using data taken with the Hubble Space Telescope. In order to measure proper motions with an expected size of several tens of milliarcseconds per century using a time baseline of 2-4 years, our work required that positions of stars and QSOs be measured to an accuracy of $\sim 0.25$ mas $(\sim 0.005$ pixel). This contribution reviews the scientific justification of this work and our methodology. It concludes with a few general results and future directions.
\end{abstract}

Keywords. astrometry, galaxies: dwarf, Local Group, methods: data analysis

\section{Introduction}

The Milky Way and Andromeda are the two most luminous and massive galaxies in the Local Group (LG). Nearly all of the remaining galaxies are dwarfs whose luminosities and masses are orders of magnitude smaller. They tend to be concentrated around the two most massive members, implying that many of them are bound satellites. This hierarchical clustering is predicted by the $\Lambda \mathrm{CDM}$ models for galaxy formation. However, there have been proposals that at least some of these satellites are the remnants of more massive progenitors tidally disrupted by the Milky Way. Lynden-Bell \& Lynden-Bell (1995) observed that some dwarf galaxies and globular clusters lie on great circles in the sky and, thus, proposed that they have similar orbits - forming streams of tidal debris. Kroupa et al. (2005) noted that 11 of the closest dwarf galaxies lie in a common plane and argue that this is inconsistent with the prediction of the $\Lambda$ CDM model at the $99.5 \%$ level (though see Kang, et al. (2005) and Zentner et al. (2005) for an opposing view).

There are nearly two dozen known dwarf galaxies within $250 \mathrm{kpc}$ of the Milky Way, with about half of them identified in the Sloan Digital Sky Survey (SDSS) data only in the past couple of years. At least a factor of two more dwarf galaxies are expected to be discovered when similar surveys are conducted in the southern hemisphere. Even if the number doubles or triples, their count would still be lower than several hundred dwarf galaxies predicted by $\Lambda \mathrm{CDM}$ simulations of the formation of the LG (Moore et al. 1999, Klypin et al. 1999).

Dwarf galaxies in the LG exhibit a wide range of properties. Their luminosities range from that of the least-luminous globular clusters to a factor of $\sim 10^{6}$ greater. Stellar populations range from being single-age and metal-poor, such as that in Draco, to having multiple ages and a spread in metallicity, such as that in Carina. Deep imaging of the 
outer regions of dwarf galaxies suggests that the Galactic tidal field has disturbed their structure. These tidal effects range from minor, as in Carina and Ursa Minor, to severe, as in Sagittarius. If the destruction and eventual incorporation of Sagittarius into the Galactic halo is not a unique event, the disruption of dwarf satellites has contributed to the formation of the Galactic stellar halo. Tidal effects may also help to explain the wide range of properties of the dwarfs.

Knowing the orbits of dwarf galaxies around the Milky Way would help to clarify some of the issues discussed above and, in particular, test models for the formation of the LG. One of the most difficult steps in obtaining an orbit is to measure the proper motion: the change in the angular position of the galaxy over time.

\section{Observational Challenge}

Measuring a proper motion, $\mu$, of a galaxy is very difficult for a number of reasons. Mathematically, $\mu=\left(46\right.$ milliarcsec century $\left.{ }^{-1}\right)\left(v_{t} / 220 \mathrm{~km} \mathrm{~s}^{-1}\right)(100 \mathrm{kpc} / d)$, where $v_{t}$ is the tangential velocity of the galaxy and $d$ is its heliocentric distance. Owing to the blurring caused by the Earth's atmosphere, time baselines of several tens of years are required to achieve a proper motion with an accuracy of about 10 mas century $^{-1}$ with groundbased telescopes. Such long time baselines create their own problems: an astrometrist must contend with images taken with different telescopes, detectors, pointings, and atmospheric conditions. Despite these challenges, measurements based on ground-based imaging exist in the peer-reviewed literature for the Fornax (Dinescu et al. 2004), LMC (e.g., Pedreros et al. 2006), Sagittarius (Dinescu et al. 2005), Sculptor (Schweitzer et al. 1995), and SMC (e.g., Kroupa \& Bastian 1997) dwarf galaxies. Measurements for a few additional dwarf galaxies are likely in the near future. Using ground-based data, reducing the uncertainties of these measured proper motions by extending time baselines is daunting and independent checks are impossible. Adaptive optics can reduce the atmospheric blurring, but it does not eliminate atmospheric refraction and the small corrected-fieldof-view makes it difficult to tie such images to the earlier data.

The launch of the Hubble Space Telescope (HST) opened up a new avenue for measuring proper motions. Freed from atmospheric effects, the resolution of HST is diffraction limited and so the required time baselines were reduced from several tens of years to only a few years. See, for example, Kallivayalil et al. (2006a) and Piatek et al. (2007). Nevertheless, there are serious scientific, engineering, and economic trade offs. The small field of view of $H S T$ means that: only a few tens to a few hundred stars enter the analysis; a single QSO per field provides the standard of rest; and corrections for the internal motions of the galaxy may be necessary. Being in space, any technical problems with the telescope or the detectors are difficult to solve and, in fact, the best onboard imager, the Advanced Camera for Surveys (ACS), is currently out of service. Operating and servicing $H S T$ is costly. Although its life will likely be extended for another decade by the next servicing mission, it is finite and there are currently no plans for a comparable replacement. In the future, space-based astrometry will face the same problems of combining data from different detectors and telescopes that complicate ground-based astrometry.

\section{Observing Method and Data Analysis}

Observational setups have varied and the reader should refer to the corresponding published article for a given galaxy. However, a typical data set has the following features. The imaged field is centered on a known QSO which serves as an extragalactic standard of rest. The accuracy of the proper motion is almost always determined by the positional 
uncertainty of the QSO. In our programs, a $S / N \simeq 100$ has achieved an accuracy of about 0.25 mas (0.005 pixel). The exposure time is typically set so that the brightest pixel in the QSO's image is about a third full, providing some protection against the variability of QSOs. Since the FWHM of a stellar PSF is comparable to the size of a pixel in the CCD, the images are dithered to reduce the effects of undersampling. Dithering also prevents the complete failure of the measurement caused by the QSO always falling on a bad pixel. Typically there are at least 8 exposures per epoch, though the actual number ranges between 4 and 40 .

We have used the WFPC2, STIS, ACS/HRC, and ACS/WFC imagers. All of these cameras introduce significant geometrical distortions which pose a problem for measuring the average motion of stars distributed throughout a field with respect to the QSO. The effect of errors in the distortion correction on the location of the QSO is minimized by using the same camera, filter, pointing, and orientation at all epochs for a given field. Unfortunately, the execution was never ideal: the pointing of the telescope could differ by several tens of pixels and the LMC and SMC programs allowed large rotations between epochs. With the demise of ACS and STIS, some of the fields are now being imaged with different detectors at different epochs. Although potentially helpful, multi-color imaging is not necessary. However, imaging in multiple fields - each centered on its own distinct QSO - is vital as this provides an independent check on the measured proper motions. Averaging the different measurements also reduces the final uncertainty.

The data analysis employed in our work has evolved over time and it has been adapted to the idiosyncrasies of particular data sets. Nevertheless, there are several canonical steps. 1) Derive the initial $(x, y)$ coordinates for the stars and the QSO in each exposure of each epoch using a standard software package. 2) At each epoch, iteratively derive a single, constant effective point-spread function (ePSF) and apply it to produce progressively more accurate $(x, y)$ coordinates of stars and the QSO for each exposure. It would be desirable to construct an ePSF that varies with position in the image for each exposure. However, our fields do not contain enough stars with sufficient $S / N$ to do so. 3) Transform the $(x, y)$ coordinates from the different exposures at a given epoch to a fiducial coordinate system and calculate the mean coordinate of every object. 4) Correct the coordinates of objects for the known geometrical distortions using available prescriptions. 5) Transform the mean coordinates of objects at the different epochs to a common coordinate system which is co-moving with the stars of the galaxy. Objects that are not members of the galaxy have a linear motion fitted in the transformation. The proper motion of the galaxy derives from the fitted motion of the QSO.

The last step above may also require correcting for the effects of the degrading charge transfer efficiency (CTE). A close inspection of images taken with HST shows "tails" behind stars, all pointing away from the serial register. They are one manifestation of the imperfect charge transfer between pixels during the readout, caused when some of the transferring charge is trapped for a period of time and then released. Since the amount of trapped charge depends on the number and distribution of "charge traps," the fractional loss decreases with increasing brightness of the star or QSO. Since the number of traps increases with time because of the damage from cosmic radiation, the effect also depends on the length of time the detector was in space. It is this time dependence that makes correcting for CTE necessary for precise astrometry. One method of correction is to "restore" images to their pre-readout state. Bristow \& Alexov (2002) developed such software for images taken with STIS; unfortunately, no comparable software exists for other detectors. When using other detectors, the correction is made by including a term depending on time, $S / N$, and distance from the serial register in the transformation between epochs. This semi-empirical correction does not capture all of the physics of 
CTE and so the authors think that a concerted effort is needed to understand CTE effects and to derive better ways of correcting them.

\section{Results and Future Work}

We have published proper motions measured with HST for the Carina, Fornax, Sculptor, and Ursa Minor galaxies. We have confirmed the Kallivayalil et al. (2006a, 2006b) measurements for the LMC and SMC, respectively. Our analysis detected the rotation of the LMC for the first time using proper motions. A paper giving a proper motion for Sagittarius derived from archival $H S T$ data is in preparation. Finally, second-epoch $H S T$ data for Draco and Sextans will have been taken by the end of 2007.

The most notable result so far from the above measurements is that orbits of the dwarf galaxies around the Milky Way tend to be polar, coplanar, and to have the same sense. However, there are exceptions. For example, the orbit of Sculptor is in the same plane but of the opposite sense. These results need to be strengthened with observations for more galaxies. Measurements for the galaxies recently discovered in the SDSS data will be particularly interesting, as they may explain the cause of their low surface brightnesses and luminosities. More measurements should also provide tighter constraints on both the shape of the Galactic potential and on our models for the formation of the LG.

Measurements for the newly-discovered galaxies will by challenging because they contain few stars and a search in the SDSS archive for bright QSOs behind them produced only a handful of possible candidates. We are searching for fainter AGN using longer exposures. An alternative approach is to use faint compact galaxies as reference points, which will necessitate much longer exposure times using HST.

The effects caused by degrading CTE will only get worse as the cameras on the HST age. We plan to develop better methods for correcting these effects. Ideally, such a software package would restore any image taken with HST given only the date of the exposure. We will also develop methods for combining data taken with different cameras, which will be increasingly more important as the HST archive grows and new cameras are installed.

\section{References}

Bristow, P. \& Alexov, A. 2002, ST-ECF Instrument Science Report CE-STIS - 2002-001

Dinescu, D. I., Girard, T. M., van Altena, W. F., \& Lopez, C. E. 2005, ApJ, 618, L25

Dinescu, D. I., Kinney, B. A., Majewski, S. R., \& Girard, T. M. 2004, AJ, 128, 687

Kallivayalil, N., van der Marel, R. P., Alcock, C., Axelrod, T., Cook, K. H., Drake, A. J., \& Geha, M. 2006a, ApJ, 638, 772

Kallivayalil, N., van der Marel, R. P., \& Alcock, C. 2006b, ApJ, 652, 1213

Kang, X., Mao, S., Gao, L., \& Jing, Y. P. 2005, $A \xi A, 437,383$

Kroupa, P. \& Bastian, U. 1997, NewA, 2, 77

Kroupa, P., Theis, C., \& Boily, C. M. 2005, A\& $A, 431,517$

Klypin, A., Kravtsov, A. V., Valenzuela, O., \& Prada, F. 1999, ApJ, 522, 82

Lynden-Bell, D. \& Lynden-Bell, R. M. 1995, MNRAS, 275, 429

Moore, B., Diemand, J., Madau, P., Zemp, M., \& Stadel, J. 2006, MNRAS, 368, 563

Pedreros, M., Costa, E., \& Mendez, R. A. 2006, AJ, 131, 146

Piatek, S. et al. 2007, AJ, 133, 818

Schweitzer, A. E., Cudworth, K. M., Majewski, S. R., \& Suntzeff, N. B. 1995, AJ, 110, 2747

Zentner, A. R., Kravtsov, A. V., Gnedin, O. Y., \& Klypin, A. A. 2005, ApJ, 629, 219 